\title{
Oral Epithelioid Hemangioendothelioma - Unusual Location of a Rare Entity
}

\author{
Alina lacob', Sebastian Comișel', Mariana Tilinca², Tibor Mezei³ ${ }^{3}$ Cecilia Petrovan \\ 1 Department of Oral and Maxillofacial Surgery, University of Medicine and Pharmacy, Tîrgu Mureş, Romania \\ 2 Department of Cellular and Molecular Biology, University of Medicine and Pharmacy, Tîrgu Mureş, Romania \\ 3 Department of Pathology, University of Medicine and Pharmacy, Tîrgu Mureş, Romania
}

\section{CORRESPONDENCE}

\section{Alina lacob}

Str. Gheorghe Marinescu nr. 38

540139 Tîrgu Mureș, Romania

Tel: +40 265215551

E-mail: czmaalina@yahoo.com

\section{ARTICLE HISTORY}

Received: November 2, 2017

Accepted: November 20, 2017
Sebastian Comișel • Str. Gheorghe Marinescu nr. 38 540139 Tîrgu Mureș, Romania. Tel: +40 265215551 Mariana Tilinca • Str. Gheorghe Marinescu nr. 38 , 540139 Tîrgu Mureș, Romania. Tel: +40 265215551 Tibor Mezei • Str. Gheorghe Marinescu nr. 38, 540139 Tîrgu Mureș, Romania. Tel: +40 265215551

Cecilia Petrovan - Str. Gheorghe Marinescu nr. 38 540139 Tîrgu Mures, Romania. Tel: +40 265215551

\begin{abstract}
Introduction: Epithelioid hemangioendothelioma is a rare, locally aggressive vascular tumor originating from soft tissue, bone, skin, and organs such as the liver or lung, exceptionally located in the oral cavity. Most of the cases of oral epithelioid hemangioendothelioma are asymptomatic, and diagnosis is hampered by the fact that the histological features are somewhat between hemangioma and angiosarcoma, with epithelioid cells, intracytoplasmic vacuoles, low mitotic activity, and (rarely) necrosis. Immunohistochemical analysis is required to rule out carcinoma or other epithelioid vascular neoplasms. Case presentation: We present a rare case of a 59-year-old Caucasian male patient with oral epithelioid hemangioendothelioma for which clinical and cytological diagnosis was difficult, in spite of the patient's history. The lesion was nonspecific, mimicking ulcerative stomatitis, but histological and immunohistochemical evaluation finally managed to establish the right diagnosis. Subsequently, the patient underwent surgical excision of the lesion followed by oncological treatment - chemotherapy. Conclusions: Although at first examination seemed that another lesion has developed, rigorous histology and immunohistochemistry tests proved the presence of epithelioid hemangioendothelioma, a very rare entity located in the oral cavity, which required a proper surgical and oncological approach.
\end{abstract}

Keywords: epithelioid hemangioendothelioma, rare oral lesions, vascular tumor

\section{INTRODUCTION}

First described as a distinctive entity in 1982 by Weiss and Enzinger, epithelioid hemangioendothelioma (EHE) is a rare malignant vascular tumor of intermediate malignant potential, originating from endothelial cells. ${ }^{1-4}$ In most cases tumor growth is slow and painless. The tumor is usually located in the soft tissue of the extremities and less frequently in the liver, lung, pleura, bones, skin, lymph nodes, and central nervous system..$^{2,5-7}$ EHE is rarely diagnosed in the head and neck region, and even more rarely in the oral cavity.,3 The World Health Organization (WHO) classifies EHE as a locally aggressive tumor with metastatic potential; the clinical and histological behavior is intermediate between hemangioma and angiosarcoma. ${ }^{1-8}$ A series of histologic types of hemangioendothe- 
lioma were described: kaposiform, Dabska-retiform (or hobnail), compound or complex, low grade polymorphic and epithelioid, the latter being considered the most aggressive type, with high metastatic potential.?

EHE is typically found as an asymptomatic solitary mass, with poorly defined infiltrative borders and painless growth. ${ }^{7}$ On microscopic examination, the epithelioid cells are usually polygonal or round, arranged in nests and cords, with wide eosinophilic cytoplasm, but spindleshape cells may be sometimes found. Tumor cells are frequently located in a fibromyxoid stroma and have round or ovoid vesicular nuclei, fine and dispersed chromatin, prominent intracytoplasmic vacuoles, and no evident nucleoli. These morphological aspects can also be found in other neoplasias (carcinomas, melanomas, other epithelioid sarcomas), thus making diagnosis very difficult. In general, cellular atypia is discreet, and the mitotic activity is low. $3,4,7,8$

The vascular phenotype can be identified with endothelial markers such as CD31, CD34, and von Willebrand factor. The latter provides high specificity with cytoplasmic marking, whereas CD31 and CD34 are more sensitive. ${ }^{4,7}$

Because of the rarity of EHE, there is no standard for treatment, and only few therapeutic options are available. ${ }^{4}$ Considering the intermediate malignant potential of EHE, the treatment of choice is surgical and consists of wide local excision of the primary tumor and close clinical followup due to the possibility of metastatic lesions and the risk for local recurrences. ${ }^{3}$

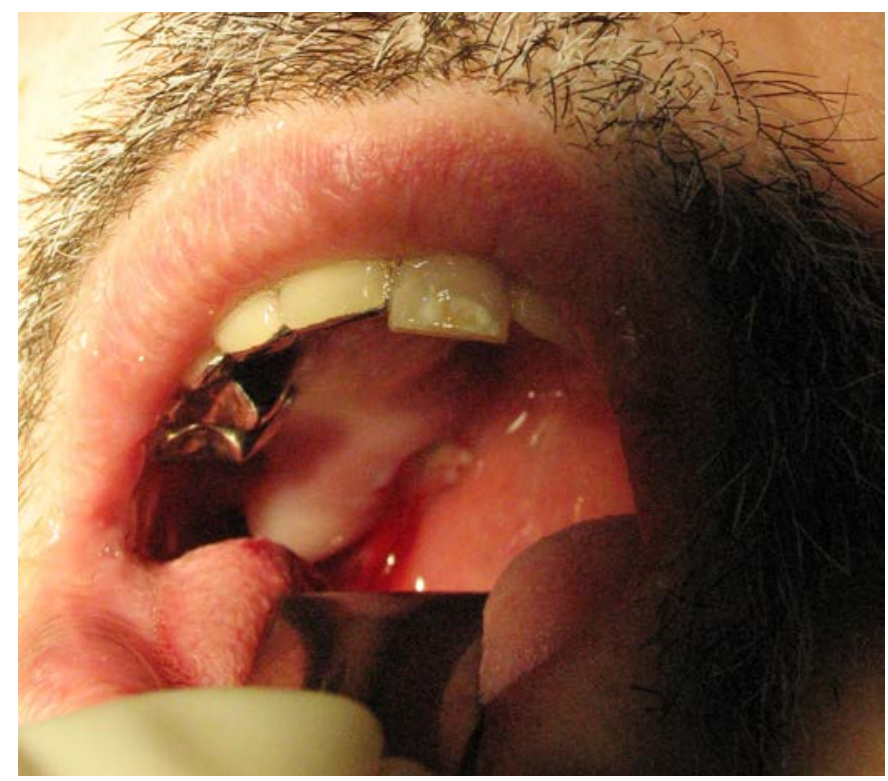

FIGURE 1. Preoperative clinical aspect: right palatal bleeding ulceration, located on a slightly protruding area
We present a case of a patient with oral epithelioid hemangioendothelioma for which clinical and cytological diagnosis was difficult, in spite of the patient's history. The lesion was quite common, imitating ulcerative stomatitis, but histological and immunohistochemical evaluation finally established the diagnosis. The patient gave informed consent allowing the publication of his data, and the institution where the patient had been admitted, approved the publication of the case.

\section{CASE PRESENTATION}

A 59-year-old Caucasian male patient presented to our department with an ulcerated, painless, progressively enlarging mass, which appeared 3-4 months before at the right side of the palate. Previously, it was associated with multiple ulcerative lesions which have healed with topical treatment. On oral examination, a $2-\mathrm{cm}$ round, slightly raised mass was found at the right posterior part of the hard palate, next to the second molar, showing a small ulcerated, hemorrhagic area on the top. The tumor had an edematous, well-defined border at the mucous membrane, without clear evidence of bone involvement. No similar lesions were identified in the oral cavity or skin, and no cervical lymph nodes were palpable. The radiographic appearance of the area was normal, without signs of bone resorption. Initial diagnosis was ulcerative stomatitis, and a topical prescription was done (Figure 1).

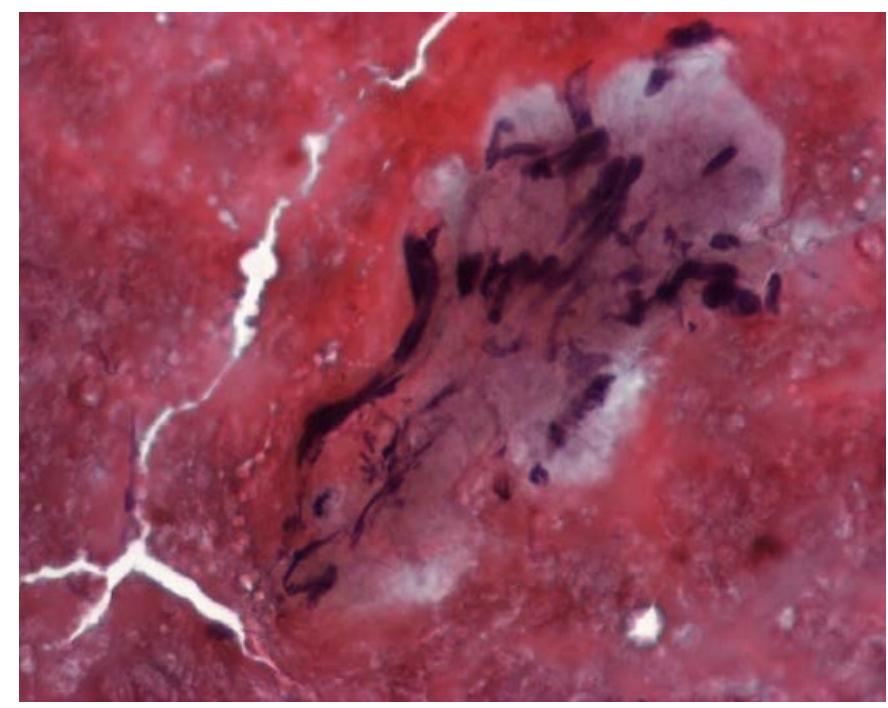

FIGURE 2. Cytological aspect: various cohesive, fusiform cells can be noted. Cytological diagnosis largely depends on the region of the tumor from which the sample was harvested, thus the aspect can often be nonspecific (ob. $\times 20$, col. HE). 
The patient's history revealed an episode of deep venous thrombosis on the right groin 10 years before, for which he received medical treatment. He was operated for a vascular tumor located in the right groin 3 years before. In the same year, he underwent D6 laminectomy with incomplete excision of an extradural tumor mass. In both situations, histology showed epithelioid hemangioendothelioma, and the patient followed chemotherapy immediately after surgical treatment (six cycles of the MAID protocol - Mesna, Doxorubicin, Ifosfamide, and Dacarbazine). The patient was followed-up with CT scans performed yearly, the last one 2 weeks before presenting in our department. These examinations indicated multiple stable tumor masses, located in the lungs, liver and vertebra, but no mention of any oral (palatal) lesion.

Fine-needle aspiration of the oral lesion was performed, but the cytological aspect was nonspecific, paucicellular with epithelial cells without atypia, numerous inflammatory cells in the background, small, uniform cells without significant pleomorphism, and rare spindle-shaped cells in a mixoid stroma (Figure 2).

Excisional biopsy was performed under local anesthesia, and the histological examination indicated multiple tumoral nodules infiltrating the salivary structures. Tumoral structures were formed by an abundant mixoid stroma with round or spindle cells with an epithelioid aspect, arranged in cords. Tumor cells were uniform, without pleomorphism, with a few mitoses and atypia, and extensive necrotic areas located in the deeper parts of the tumor

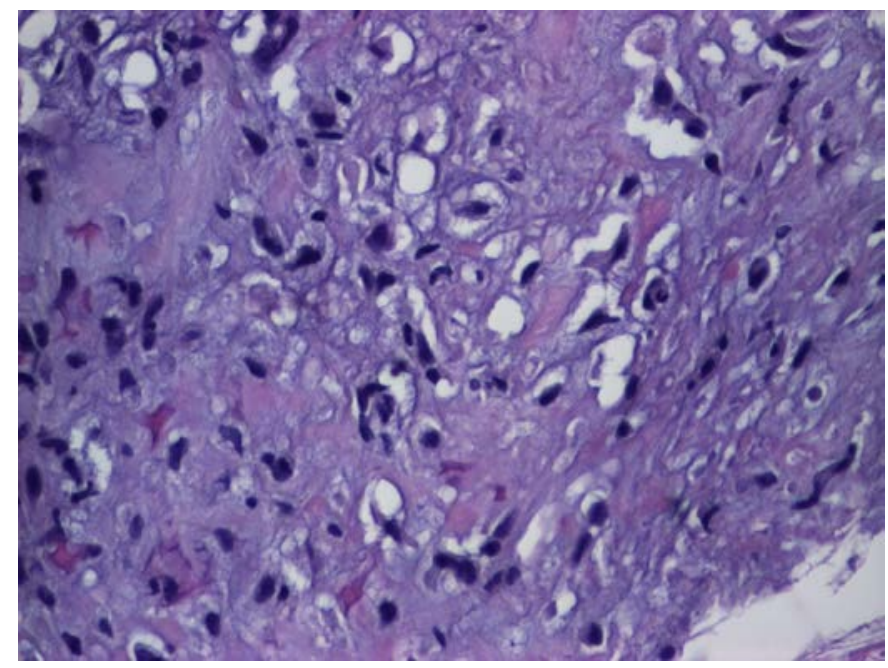

FIGURE 3. Histological aspect of the epithelioid hemangioendothelioma: proliferation of epithelial endothelial cells of different sizes and various shapes, some spindle-shaped with eosinophilic cytoplasm, with patches of vacuolar cytoplasm. Some of the cells show intracytoplasmic lumens (ob. $\times 20$, col. HE).
(Figure 3). On immunohistochemical examination, CD31 and CD34 staining confirmed the vascular endothelial nature of the tumor, and there was also a positive reaction for Factor VIII and cytokeratins AE1/AE3; however, PS100 was found negative.

The patient underwent wide local excision of the right palatal mass and the underlying bone under general anesthesia, followed by the placement of a palatal prosthesis in order to protect the healing process and to observe possible local recurrences. Surgical treatment was limited to tumor excision, without extensive reconstructive procedures due to the presence of multiple tumor locations and the high risk of local recurrence, but also taking into consideration the patient's option (Figure 4). Afterwards, the patient restarted chemotherapy - five cycles of Taxotere followed by Vinorelbine, which was changed with Gemcitabine due to digestive toxicity. He also underwent symptomatic radiotherapy into the $\mathrm{D} 6, \mathrm{D} 7$, and D8 vertebral area using a 30Gy total dose in $2 \mathrm{~Gy}$ daily fractions. The patient died after two years as a result of disease progression, but without any signs of recurrence in oral cavity.

\section{DISCUSSIONS}

Although intraoral EHE has been described earlier, the literature is difficult to assess because the lesion received various names over time. Most reported cases were clinically diagnosed as benign entities including pyogenic granuloma, fibroma, peripheral giant cell granuloma, peripheral

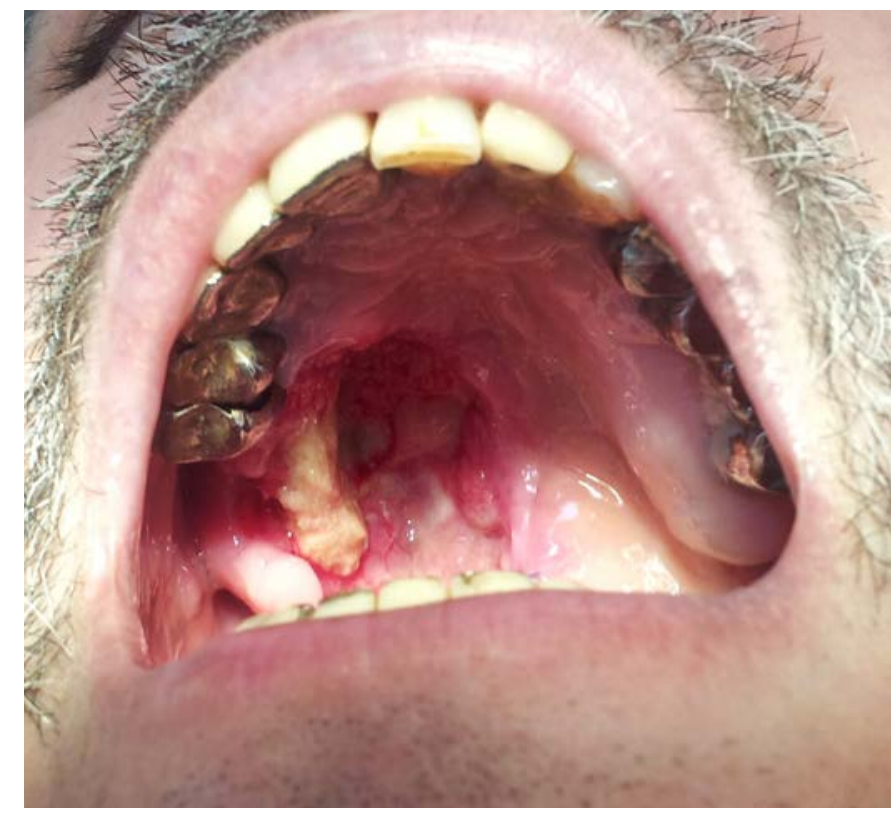

FIGURE 4. Postoperative aspect (7 days after surgery) 
ossifying fibroma, inflammatory fibrous hyperplasia, and necrotizing ulcerative gingivitis. ${ }^{2,3,9}$ The case reported here is similar to those reported earlier in the literature, as the initial hypothesized clinical diagnosis was that of ulcerative gingivitis. The great number of EHE that are clinically diagnosed as benign lesions can be quite problematic, since it is a type of neoplasm with malignant biological behavior that is intermediate between hemangioma and conventional angiosarcoma, with reported cases with aggressive behavior and multiple local recurrences. ${ }^{2,3}$

The clinical features of EHE include a solitary mass localized either superficial or deep, asymptomatic, with poorly delimited contour, infiltrating the adjacent structures. ${ }^{5,8}$ Sometimes the tumor may show multifocal or metastatic behavior. The case presented here was painless, with no important complaint except for a slight deformation of the palate and persistent 'ulcerative stomatitis'.

Radiological investigations are usually nonspecific, showing a poorly defined osteolytic lesion, with resorption or destruction of the underlying bone. ${ }^{3}$ Our patient had no radiographic changes either on orthopantomography or CT scan.

So far, the pathogenicity of EHE has not been totally elucidated. For some authors the distinction among hemangiendotheliomas is controversial, since they believe these lesions represent a sub-type of low grade angiosarcoma, which is less cellular, less pleomorphic, and it has lower mitotic activity. ${ }^{7,10}$

There are no clear criteria for predicting the biologic behavior of EHE; however, it was indicated that the presence of an increased number of mitoses, significant cellular atypia, high proportion of spindle cells, areas of necrosis, and metaplastic bone formation can be considered morphological signs of tumors with a more aggressive behavior and more reserved prognosis, associated with a high risk of recurrences and regional or distant metastases. ${ }^{3}$ In our patient, histological assessment highlighted spindle cells and extensive areas of necrosis, but only a few mitoses and discrete cellular atypia, suggesting that the lesion had intermediate aggressiveness.

On cytological examination, EHE usually presents with round or oval epithelioid cells with moderate cytoplasm and nuclei with moderate pleomorphism, rare mitosis, and sometimes pseudo-inclusions, ${ }^{11}$ but in our case the cytology was nonspecific, describing only the cells on the smear, without indicating a diagnosis.

As the morphological characteristics of EHE are nonspecific, the lesion can be confused with many others, including hemangioma, squamous cell carcinoma, or melanoma. ${ }^{3}$ Immunohistochemical tests are important for establishing the diagnosis, with the majority of intraoral EHE lesions being immunoreactive to CD34, CD31, factor VIII-RAg, and vimentin, highlighting the epithelioid endothelial origin of the entity. ${ }^{3-5,7,12}$ The samples obtained from our patient showed immunoreactivity to CD31, CD34, and factor VIII, and also for cytokeratin AE-1/AE-3 (this latter aspect might create confusion with squamous cell carcinoma).

Recent molecular studies demonstrated a consistent genetic abnormality - $\mathrm{t}(1,3)(\mathrm{p} 36.23$; q25.1) - in EHE of various anatomic sites. The translocation fuses CAMTA1 on 1p36.23 to WWTR1 on 3q25.1 and is particular for EHE, being helpful as a molecular diagnostic tool in challenging situations. ${ }^{13}$

A tumor with uncertain biological behavior, EHE can present local aggressive behavior in some cases, with possible distant metastases, while in other cases the clinical evolution might be painless, with healing after complete surgical resection. The rarity of this disease hinders the development of standardized therapeutic protocols; therefore, management and follow-up are to be established on an individual basis for each patient. ${ }^{7}$ Wide local excision was chosen as the therapeutic option in the majority of cases reported in the literature, because of the intermediate malignant potential of the EHE; ${ }^{3}$ also, conservative procedures may favor the local recurrence of the lesions. Oncologic therapy (chemotherapy, radiotherapy) were suggested for the treatment of EHE, but with unsatisfactory results. ${ }^{3,12}$

The evolution and prognosis of EHE are intermediate between those of hemangioma and angiosarcoma, and often remain unpredictable, unrelated to microscopic findings. The tumor's aggressiveness is given by the high rates of metastasis (20-30\%) and mortality (10-20\%), especially when it affects soft tissues and organs. $3,7,8,12$ Reported cases have emphasized that lesions located in the upper gingiva and oral mucosa are the ones with most relapses. ${ }^{3,7}$

Clinical patterns with prognostic significance in EHE have been investigated in a recent internet registry study comprising a large group of patients. No difference was found in survival rate based on the presence of metastatic disease or single organ versus multiple organ involvement. ${ }^{14}$

Based on our patient's medical history, clinical and radiological data, and especially the histological aspects, we considered the case as an advanced form of multifocal epithelioid hemangioendothelioma with slow development. One of the lesions appeared intraorally, mimicking a benign lesion, and thus it was initially difficult to establish a proper diagnosis. After surgical treatment, the patient un- 
derwent complex oncological treatment (chemotherapy and radiotherapy). The current concept is that separate lesions are independent primary tumors rather than metastases. ${ }^{15}$

\section{CONCLUSIONS}

The physician should bear in mind the possibility of rare lesion development when the oral cavity is examined. These lesions require careful histopathological and immunohistochemical evaluation to establish the right diagnosis. Epithelioid hemangioendothelioma is extremely rarely located in the oral cavity and needs various diagnostic techniques to be recognized and treated properly, especially considering its malignant biological behavior.

\section{CONFLICT OF INTEREST}

Nothing to declare.

\section{REFERENCES}

1. Bioulac-Sage P, Laumonier H, Laurent C, Blanc JF, Balabaud C. Benign and Malignant Vascular Tumors of the Liver in Adults. Semin Liver Dis. 2008:28:302-314

2. Mohtasham N, Kharrazi AA, Jamshidi S, Jafarzadeh $H$. Epithelioid hemangioendothelioma of the oral cavity: a case report. J Oral Maxillofac Surg. 2008;50:219-223.

3. Gordón-Núñez MA, Silva LM, Lopes MF, de Oliveira-Neto SF, Maia AP, Galvão HC. Intraoral epithelioid hemangioendothelioma: A case report and review of the literature. Med Oral Patol Oral Cir Bucal. 2010;15:e340-e346.
4. Sardaro A, Bardoscia L, Fonte Petruzzelli M, Portaluri M. Epithelioid hemangioendothelioma: an overview and update on a rare vascular tumor. Oncol Rev. 2014;8:259.

5. Lakshmi SV, Prabhavathy D, Jayakumar S, Janaki C, Tharini GK. Epithelioid hemangioendothelioma: A rare vascular tumor. Indian J Dermatol. 2012;57:53-54

6. Flucke U, Vogels RJ, de Saint Aubain Somerhausen N, et al. Epithelioid Hemangioendothelioma: clinicopathologic, immunhistochemical, and molecular genetic analysis of 39 cases. Diagn Pathol. 2014;9:131.

7. Araújo Cavalcanti de Albuquerque AK, de Oliveira Romano S, Amaral Eisenberg AL. Epithelioid hemangioendothelioma: 15 years at the National Cancer Institute. Literature review. Jornal Brasileiro de Patologia e Medicina Laboratorial. 2013;49:119-125.

8. Fletcher CDM, Unni KK, Mertens F, editors. World Health Organization Classification of Tumours. Pathology and Genetics of Tumours of Soft Tissue and Bone. Lyon, France: IARC Press; 2002.

9. Molina Palma MI, Cervantes Góngora JA, García de la Torre E, Conde Pérez de la Blanca I, Ramírez Tortosa CL. Primary intraoral epithelioid hemangioendothelioma. Case report and review of the literature. Acto Otorrinolaringol Esp. 2002;53:215-218.

10. Kösemehmetoglu K, Gedikoglu G, Ruacan S. Morphological and immunohistochemical features of malignant vascular tumors with specia emphasis on GLUT1 and FKBP12 expressions. Turk Patoloji Derg. 2011;27:57-67.

11. Murali R, Zarka M, Ocal I, Tazelaar H. Cytologic Features of Epithelioid Hemangioendothelioma. Am J Clin Pathol. 2011;136:739-746.

12. Folpe AL. Soft-Tissue Tumors of the Head and Neck. In Gnepp DR, ed. Diagnostic Surgical Pathology of the Head and Neck, 2nd ed, Saunders, Philadelphia. 2009; p. 647-728.

13. Errani $C$, Zhang L, Sung YS, et al. A novel WWTR1-CAMTA1 gene fusion is a consistent abnormality in epithelioid hemangioendothelioma of different anatomic sites. Genes Chromosomes Cancer. 2011;50:644-653.

14. Lau K, Massad M, Pollak C, et al. Clinical patterns and outcome in epithelioid hemangioendothelioma with or without pulmonary involvement: insights from an internet registry in the study of a rare cancer. Chest. 2011;140:13121318.

15. Errani C, Sung YS, Zhang L, Healey JH, Antonescu CR. Monoclonality of multifocal epithelioid hemangioendothelioma of the liver by analysis of WWTR1-CAMTA1 breakpoints. Cancer Genetics. 2012;205:12-17. 